\title{
Atomic Scale Debye-Waller Thermometry
}

\author{
Menglin Zhu ${ }^{1}$, Jared Johnson ${ }^{1}$ and Jinwoo Hwang ${ }^{1}$ \\ 1. Department of Materials Science and Engineering, The Ohio State University, Columbus, OH, USA.
}

High angle annular dark field (HAADF) signal in scanning transmission electron microscopy (STEM) primarily detects the thermal diffuse scattering by phonon, and therefore it is sensitive to the temperature of the material that is being probed [1]. Since HAADF-STEM can achieve atomic resolution, one would then be able to quantify the attenuation of the HAADF intensity as a function of temperature, also known as Debye-Waller factor, of each atomic column of a crystalline material to obtain the temperature information with resolution close to the atomic scale (Figure 1a to 1c). This atomic scale Debye-Waller thermometry can enable, for example, the measurement of thermal transport characteristics within very localized volumes of the material (e.g. nanoscale regions near crystallographic defects). Such information would be important for thermal engineering of novel materials and devices, but it has been unobtainable because of the low spatial resolution of the currently existing thermal measurement techniques, including Raman spectroscopy, time-domain thermo-reflectance, or even electron energy loss spectroscopy [2], all of which have effective spatial resolution of $\sim 100$ nanometers or lower.

We advance the state-of-the-art in situ quantitative STEM experiment and analysis [3] to realize the unprecedented atomic scale Debye-Waller thermometry (Figure 1d to 1f). This new technique requires the use of a low electron dose by fast scanning of the probe over the sample with a short dwell time to suppress any extra vibration that the probe may induce to the lattice [4]. This is important, because such extra vibration of the lattice will generate background intensity in the HAADF data and complicate the analysis. A low electron dose also helps suppress any radiation damage (e.g. knock-on damage) to the specimen, and greatly reduces the negative effects of sample drift and scan noise.

The problem of using a low electron dose, however, is the low signal-to-noise ratio. We overcome this issue using non-rigid registration of fast-scanned images [5]. The method effectively increases the probe dwell time, which substantially improve the signal-to-noise ratio and the precision in scattering intensity. To demonstrate this, we have taken up to 23 fast-scanned images of a sample area, and then different numbers of images were registered to show the effect. Figure $2 \mathrm{a}$ shows that the registration with 23 images will provide higher precision and lower uncertainty than the one fast-scanned image. Here, uncertainty means how much each pixel value changes on average, in terms of standard deviation, between two separately taken images of the same sample area. Experimental uncertainty decreases to about $0.8 \%$ after registration using 23 images. Exponential fit to the data predicts that uncertainty will decrease further to about $0.4 \%$ with 30 images, and $0.13 \%$ with 50 images.

The high precision in the data discussed above directly affects the temperature resolution of the DebyeWaller thermometry. Figure $2 \mathrm{~b}$ shows a map of $\Delta T$, minimum detectable temperature difference, as a function of $T$, the upper bound temperature of $\Delta T$, and the (experimental) uncertainty, calculated based on the simulated atomic column intensity data for $\mathrm{SrTiO}_{3}$ shown in Figure 1a. This map can be used as the guide to set the optics and temperature in situ. For example, the $T$ of $\sim 600 \mathrm{~K}$ and the experimental uncertainty $\sim 0.3 \%$, which is achievable using the non-rigid registration (Figure $2 \mathrm{a}$ ), will be able to detect $\Delta T \sim 3 \mathrm{~K}$. The map can be further optimized based on the materials used, since each material has different range for thermal diffused scattering [6]. 
References:

[1] DA Muller et al., Ultramicroscopy 86 (2001), p. 371.

[2] OL Krivanek et al., Nature 514 (2014), p. 209.

[3] J Hwang et al., Phys. Rev. Lett. 111 (2013), p. 266101.

[4] A Rezikyan et al., Microsc. Microanal. 21 (2015), p. 1455.

[5] AB Yankovich et al., Nat. Commun. 5 (2014), p. 4155.

[6] This work is supported by NSF CAREER Program, DMR-1847964.
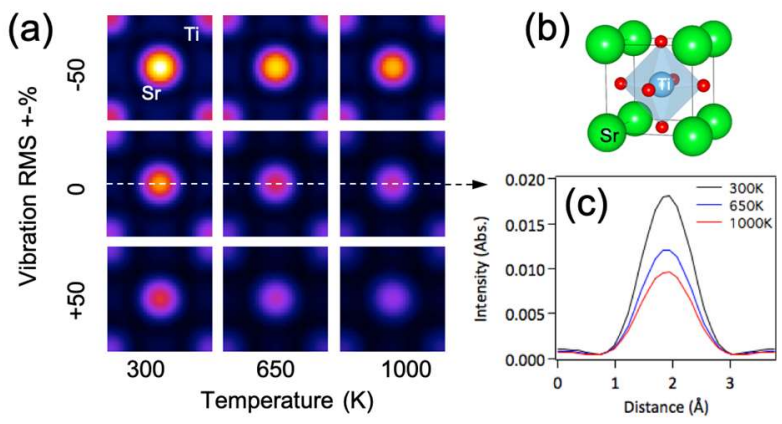

(d)

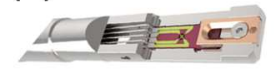

(e)

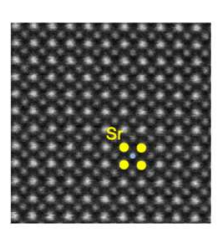

(f)

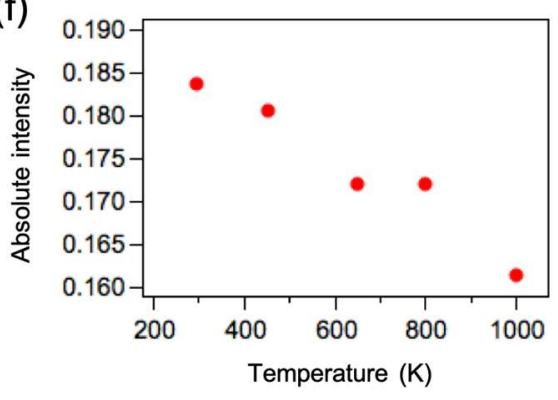

Figure 1. (a) Simulated HAADF images of the $\mathrm{SrTiO}_{3}$ unit cell shown in (b), as a function of temperature and the vibration RMS value deviated from the normal value. (c) Intensity profiles of the $\mathrm{Sr}$ column shown in (a) for different temperatures. (d) DENS Wildfire in situ thermal holder used for this study. (e) Experimental HAADF image of $\mathrm{SrTiO}_{3}$. (f) The absolute intensity of $\mathrm{Sr}$ column as a function of the temperature controlled by the in situ holder.

(a)

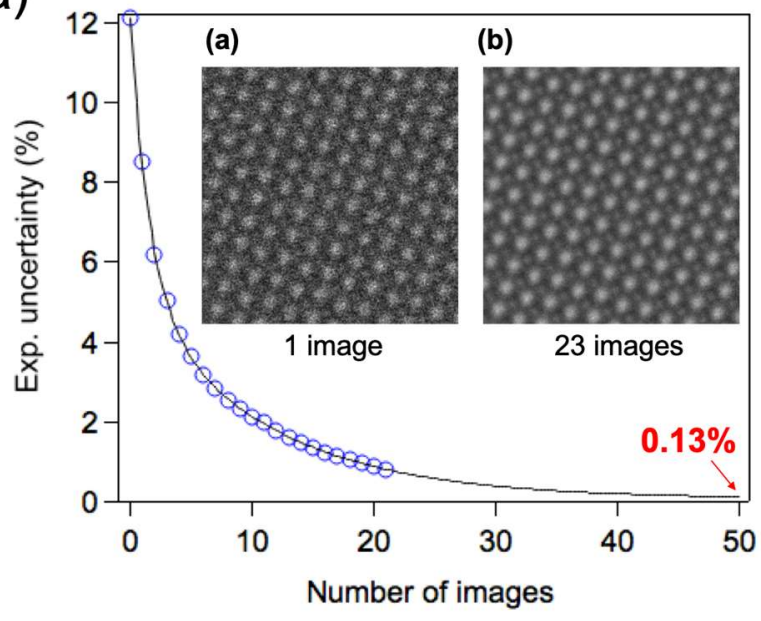

(b)

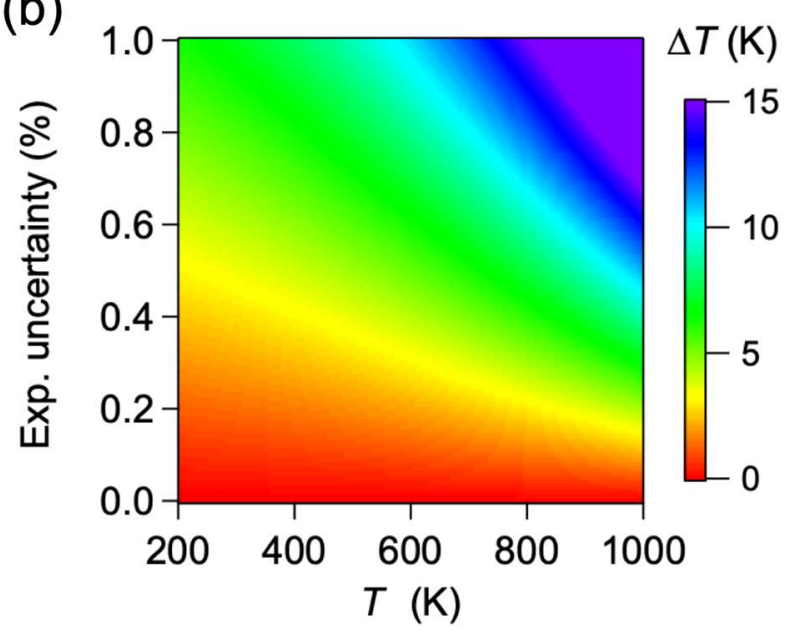

Figure 2. (a) Experimental error (\%) measured from the HAADF images, as a function of the number of images aligned using non-rigid registration. Insets show (a) one and (b) 23 fast-scanned STEM images. (b) Calculated map of the detectable $\Delta T$ range as a function of temperature $(T)$ and the uncertainty, for the HAADF range of $\mathrm{SrTiO}_{3}, 80-320 \mathrm{mrad}$. 